\title{
Shock Waves in Nanomechanical Resonators
}

\author{
Florian W. Beil \\ Center for NanoScience, Ludwigs-Maximilians-Universität-München, Geschwister-Scholl-Platz 1, 80539 München, Germany \\ Achim Wixforth \\ Lehrstuhl für Experimentalphysik I, Universität Augsburg, Universitätsstraße 1, D-86135 Augsburg, Germany
}

Werner Wegscheider and Dieter Schuh

Institut für Angewandte und Experimentelle Physik, Universität Regensburg, 93040 Regensburg, Germany

\author{
Max Bichler \\ Walter Schottky Institut, Am Coloumbwall 3, 85748 Garching, Germany
}

\begin{abstract}
Robert H. Blick*
Electrical and Computer Engineering, University of Wisconsin-Madison, 1415 Engineering Drive, Madison, Wisconsin 53706, USA
\end{abstract}

(Received 21 July 2007; published 15 January 2008)

\begin{abstract}
We study the formation of shock waves in a nanomechanical resonator with an embedded twodimensional electron gas using surface acoustic waves. The mechanical displacement of the nanoresonator is read out via the induced acoustoelectric current. Applying acoustical standing waves, we are able to determine the so-called anomalous acoustocurrent. This current is found only in the regime of shock wave formation.
\end{abstract}

DOI: 10.1103/PhysRevLett.100.026801

PACS numbers: 73.50.Rb, 47.40.-x, 63.20.K-

The dream of every surfer is an extremely steep wave propagating at the highest speed possible. The best waves for this would be shock waves, but they are very hard to surf. In the nanoscopic world the same is true: the surfers in this case are electrons riding through nanomechanical devices on acoustic waves [1]. Naturally, this is of interest for fundamental aspects of nanoelectromechanical systems, when it comes to quantum limited displacement detection [2] and the control of phonon number states [3,4].

Over the past two decades a multitude of work focused on reducing the dimensionality of electronic systems from three to zero dimensions [5]. Only recently the availability of even more intricate nanostructuring techniques made it possible to morph the dimensionality of the phonon systems, i.e., to construct phonon cavities with nanoscale dimensions. An example of this are freely suspended two-dimensional electron gases (2DEG), for which the phonon modes can be tailored [6]. This allows studies of dissipation in the limit of single electrons interacting with discrete phonon modes [7], which is revealed in the formation of van Hove singularities in the phonon density of states for low-dimensional phonon systems [8]. Furthermore, suspended 2DEGs, quantum wires, and quantum dots are the perfect tools for studying quantum electromechanical effects [9] and might further improve measurements for the current standard [10].

The main limitation encountered so far is the fact that the phonon system, in contrast to the electronic one, could not be actuated directly. Nevertheless, the method of choice for generating such an acoustic actuation is readily available: surface acoustic waves (SAW) are a proven tool for investigating 2DEGs [11]. Hence, we set out to combine 2DEGs embedded in a nanomechanical resonator with SAWs generated by interdigitated transducers (IDTs). In early work we demonstrated how to achieve acoustical coupling of a nanomechanical resonator (suspended beam without 2DEG) to SAWs [12,13]. Recently, we were able to resolve single defect relaxation [14] and phonon population inversion in nanoresonators [15].

In this Letter, we present acoustical excitation of a nanoresonator with an integrated 2DEG by SAW, leading to the formation of shock waves in the nanomechanical system. Shock wave formation is probed via the anomalous acoustoelectric effect, one of the manifestations of the electronphonon interaction. Acoustoelectric effects have up to now been mainly studied in quantum wells, where the mechanical properties are determined by the bulk $[11,16]$. It is apparent that the investigation of acoustoelectric effects in suspended 2DEGs is also interesting for improving current standards $[10,17,18]$ and elucidating effects like the anomalous acoustoelectric effect as being observed in thin films [19]. The SAW induced current directly traces the shock wave formation in the nanoresonator.

The processing follows standard techniques for suspended 2DEGs [20,21], with the difference that we integrate IDTs for SAW generation. In Fig. 1 a scanning electron micrograph of the sample is shown. The inset presents the suspended beam of length $L=1.2 \mu \mathrm{m}$, width $w=300 \mathrm{~nm}$, and height $h=200 \mathrm{~nm}$ used in the experiments. As seen, the suspended sample is placed between 


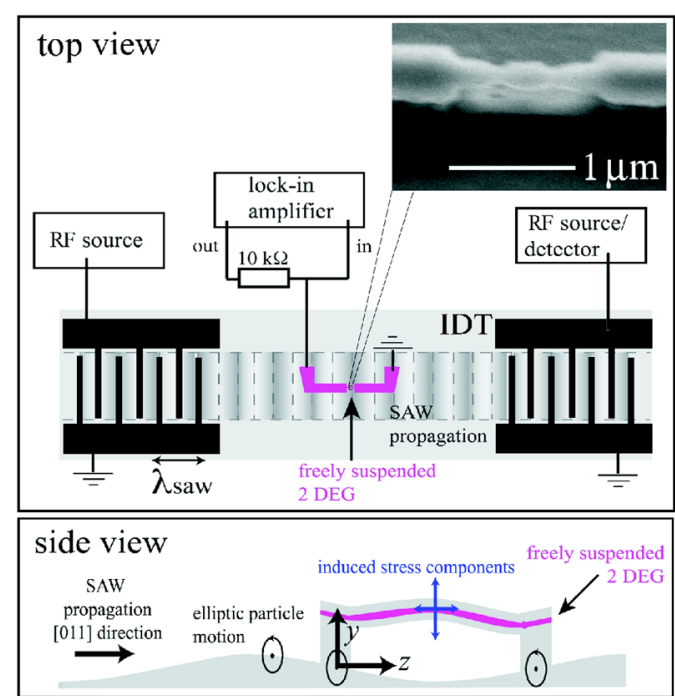

FIG. 1 (color online). Top and side views of the sample geometry and experimental setup. The micrograph of the sample shows the suspended specimen placed in the delay line formed by IDTs. The suspended 2DEG interacts with the traveling SAW via the elliptic motion of clamping points, which induces acoustoelectric currents.

two IDTs forming an acoustic delay line. For the first set of experiments, we generate the acoustic waves in one transducer, while for shock wave probing we couple both IDTs to generate an acoustical standing wave pattern and then trace the induced direct current. The transducers generate a coherent acoustic sound wave via the inverse piezo effect, at the lithographically defined center frequency $f_{\text {saw }}$. This corresponds to a SAW wavelength $\lambda_{\text {saw }}=7 \mu \mathrm{m}(9 \mu \mathrm{m})$. The SAW frequency and wavelength are connected via $f_{\text {saw }}=v_{\text {saw }} / \lambda_{\text {saw }}$, where $v_{\text {saw }}=2865 \mathrm{~m} / \mathrm{s}$ is the surface wave velocity on GaAs in the [011] direction. In order to measure the acoustoelectric effects in the 2DEG in a two point fashion, we either employ a lock-in technique or measure the direct current driven through the 2DEG (see Fig. 1).

For determining the quality of the suspended electron system, we first took magnetoresistance traces of the suspended 2DEG at $100 \mathrm{mK}$ [Fig. 2(a)]. The longitudinal resistance exhibits $1 / B$ periodic Shubnikov-de Haas oscillations, from which we calculate a carrier density of $6.56 \times 10^{15} \mathrm{~m}^{-2}$ with a mobility $3057 \mathrm{~cm}^{2} /(\mathrm{V} \mathrm{s})$. The peak in the resistance at low magnetic field is due to coherent backscattering effects in the suspended 2DEG [20]. The electron gas was tuned into high resistance by applying a backgate voltage $\left(V_{\mathrm{bg}} \approx 200 \mathrm{~V}\right)$. In Fig. 2(b), we show the effect of acoustic excitation by the left and right IDT firing at the suspended beam. Scanning the radio frequency signal applied to the left IDT results in a modulation of the current. A notable current modulation is observed only when $f \sim f_{\text {saw }}$ and a maximal acoustic power is generated.

In detail, the induced acoustoelectric current occurs only when either the left or the right IDT is driven by one of the
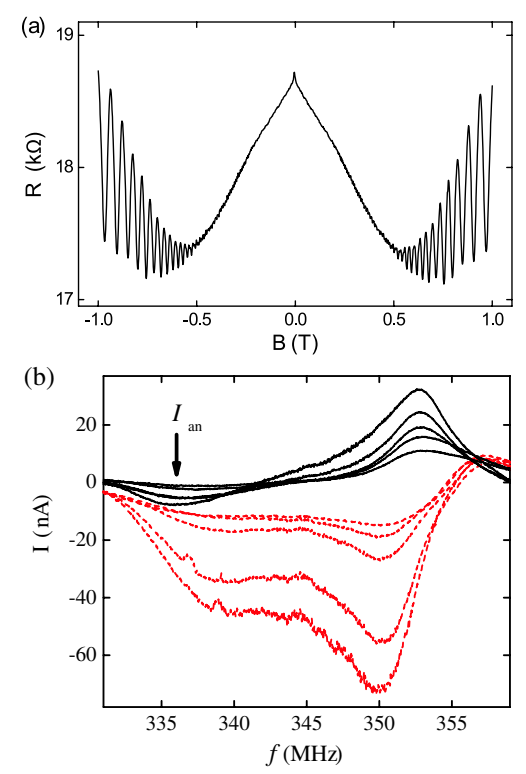

FIG. 2 (color online). (a) Longitudinal magnetoresistance with Shubnikov-de Haas oscillations well pronounced. (b) Acoustoelectric current at $1.5 \mathrm{~K}$ with surface acoustic waves driving the current from the left (black) and right (red) (source-drain voltage $V_{\mathrm{DS}}=50 \mu \mathrm{V}$. At $f=f_{\text {saw }}$, the IDTs generate acoustical waves and the acoustoelectric current is observed as a dip in the current. Driving an acoustic excitation from the right reverses the sign of the normal acoustoelectrical current $I_{n}$ (traces are for power levels from $0 \mathrm{dBm}$ to $10 \mathrm{dBm}$ ). The remarkable feature is the region in the shoulder of the acoustic bandpass: for both directions of the acoustic excitation the anomalous acoustoelectric current $I_{\text {an }}$ shows no sign reversal.

synthesizers. While the left transducer generates a forward current, the right one reverses the current direction and pumps electrons backwards. These traces are recorded under increasing rf power. The dependence of the SAW induced current on IDT frequency $f$, SAW power, and propagation direction are consistent with previous measurements of the acoustoelectric current in nonsuspended 2DEGs [17] and quantum point contacts [18]. The amplitude of the normal acoustoelectric current is given by $I_{n}=$ $n e f_{\text {saw }}$, where $n$ is the integer number of electrons transferred: this suggests that during each SAW cycle $\sim 380$ electrons are transferred.

A peculiar feature appears in Fig. 2(b) at the left edge of the IDTs' main transmission centered at $350 \mathrm{MHz}$ : the acoustic current in the shoulder of the current peak is caused by an additional current that is not changing sign with SAW direction. This anomalous acoustoelectric current $I_{\text {an }}$ was first observed in thin manganite films [19] and was explained by the elastic deformation of the thin film by the SAW. The resulting unidirectional current $\left(I_{\text {an }}\right)$ is found to be even in the SAW wave vector $k_{\text {saw }}$, and thus invariant under inversion of SAW direction. The total acoustoelectric current then is given as a superposition of the normal and anomalous components $I_{n}+I_{\mathrm{an}}$.

It is this anomalous acoustoelectric current that allows us to probe the deformation of the nanomechanical device 
directly. In order to read out the unidirectional current, we conducted acoustic standing wave experiments: a standing wave is formed by applying phase locked rf signals to both IDTs. Shifting the relative phase $\phi$ of the driving signal at one IDT with respect to the other results in a lateral shift of the standing wave pattern. If a perfect standing wave is formed, the total wave vector equals zero and the only contribution to the measured acoustoelectric current is the anomalous component $I_{\text {an }}$. In other words, the normal acoustoelectric current depends only on the propagating part, whereas the anomalous current depends on the mechanical deformation induced in the suspended 2DEG. Thus we have a direct relation between the mechanical deformation and the relative phase $\phi$, which allows us to map the mechanical mode of the suspended beam.

In Fig. 3(a) the standing wave pattern of the anomalous current is shown: evidently the initial trace with moderate power levels applied to the IDTs is a fundamental mode of the suspended nanomechanical device, forming a sinusoidal-trace. Increasing the acoustic power leads to a large current. Evidently, the shape of the standing wave pattern is changed once the relative phase $\phi$ of the two synthesizers is altered. The deviation from the sinusoidal wave form shows up as a pronounced peak around $\phi=$ $180^{\circ}$. This is the transition from linear to nonlinear response, where the steepening of the trace indicates shock wave formation. By varying $\phi$, we are mapping the deformation of the resonator directly in the current response. In Fig. 3(b), the standing wave pattern of the anomalous current is shown for even larger acoustic excitation power levels: the shock wave form now develops extremely high current levels around $\phi=0^{\circ}$ and $360^{\circ}$. A pronounced peak at $\phi=180^{\circ}$ is accompanied by two apparent shoulders to its left and right. For the highest power levels shown in the figure, the "wave form" returns to one resembling a sinusoidal again. In Fig. 4(a), we show the full power dependence of the acoustic current while altering $\phi$ from smallest to largest driving powers at the IDTs. As seen, the ground mode shows a slight sinusoidal modulation that deforms into a shock wave, indicated by the sharp peak and the two shoulders discussed above at intermediate power levels. Evidently, this series of traces depicts a transition from the first fundamental mode to the next higher mode with a larger current amplitude.

To model this effect, we calculated the anomalous acoustoelectric current in the suspended 2DEG, following Ref. [19]. When an acoustic wave interacts with a thin film, the induced mechanical strain modulates the local conductivity, which induces an acoustoelectric current density $j$ per unit length

$$
j(z)=\frac{a \omega}{2 \pi} \int_{0}^{2 \pi / \omega} \sigma_{z z}(z, t) E_{z}(z, t) d t,
$$

where $\omega$ is the SAW frequency, $a$ the thickness of the resonator, $\sigma_{z z}$ is the component of the conductivity tensor along the $z$ axes (the [110] direction; cf. Fig. 1), and $E_{z}$ is
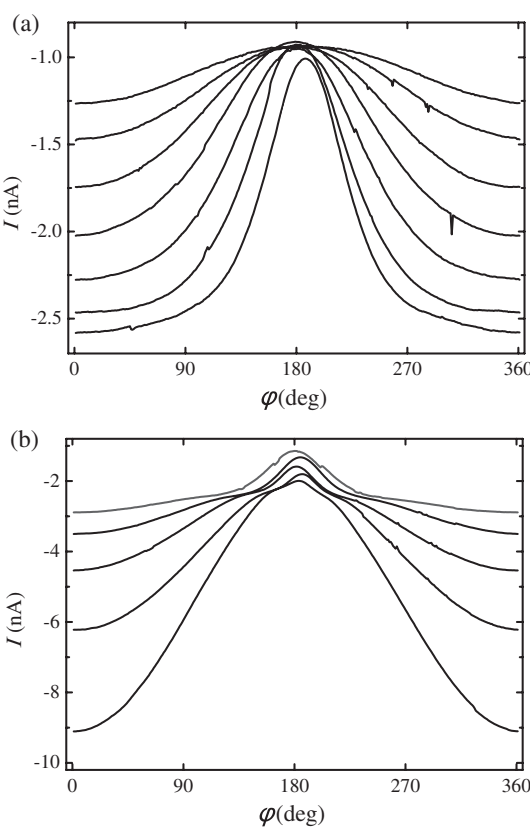

FIG. 3. (a) Standing wave measurements-nonlinear response: the current, sensitive to the relative phase shift $\phi$, is induced by the acoustic standing wave proportional only to the anomalous acoustoelectric current. The maximum current appears at $180^{\circ}$ phase shift. The applied rf power at the IDTs increases from $-40 \mathrm{dbm}$ to $-10 \mathrm{dBm}$. (b) The sinusoidal shape evolves into a $(\sin x) / x$ shape, indicating the shock front. At larger powers the resonator jumps into the next higher acoustic mode and the sinusoidal wave form reemerges.

the electric field along $z$. The SAW induced strain in the beam $S_{i j}$ will modulate the conductivity following

$$
\begin{aligned}
\sigma_{z z}(z, t)= & \sigma_{0}\left[\Pi_{z z z z} S_{z z}(z, t)+\Pi_{z z y y} S_{y y}(z, t)\right. \\
& \left.+\Pi_{z z z y} S_{y z}(z, t)\right],
\end{aligned}
$$

where $\sigma_{0}$ is the unperturbed conductivity, and the tensor $\Pi_{i j k l}$ describes the effect of strain $S_{j k}$ on the conductivity $\sigma_{i k}$ and evaluates to $\Pi_{i j k l}=\delta \sigma_{i j} / \delta S_{j k}$. To solve Eq. (1) in combination with Eq. (2), we have to determine the SAW induced strain in the beam and relate it to $E_{z}$. If two counterpropagating SAWs form a standing wave pattern, the motion of the clamping points will induce stress in the beam, extending from 0 to $L$ in the $z$ direction, which is calculated to [14]

$$
\begin{aligned}
& S_{z z}(z, t)=\frac{2 A_{l}\left[\cos (\phi / 2)+\cos \left(k_{\mathrm{saw}} L+\phi / 2\right)\right]}{L}, \\
& S_{z y}(z, t)=2 A_{t}\left(\frac{6 z^{2}}{L^{3}}-\frac{6 z}{L^{2}}\right)\left[\cos (\phi / 2)-\cos \left(k_{\mathrm{saw}} L+\phi / 2\right)\right],
\end{aligned}
$$

where $A_{t}\left(A_{l}\right)$ are the transversal (longitudinal) components of the SAW motion, and $L$ is the length of the beam. The relation between the strain and the electric field is found in the piezoelectric constitutive relation $D_{z}=$ $\epsilon_{\mathrm{GaAs}} E_{z}+e_{z 4} S_{z y}$, where $D_{z}$ is the electric displacement 

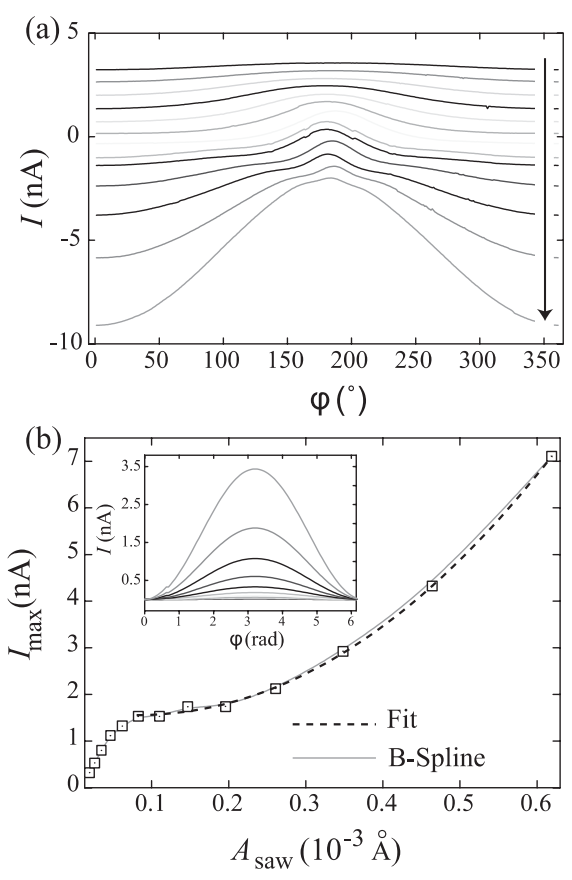

FIG. 4. (a) Full scan: At large powers the resonator jumps into the next higher acoustic mode and the sinusoidal wave form reemerges. (b) Maximum current vs SAW amplitude and comparison to model calculations.

in $z$ direction, $\epsilon_{\mathrm{GaAs}}$ is the dielectric constant of GaAs, and $e_{z 4}$ is the appropriate constant of the piezoelectric tensor, which has to be taken in the [110] coordinate system. This can be solved for $E_{z}$ to give

$$
E_{z}(z, t)=-\frac{e_{z 4}}{\epsilon_{\mathrm{GaAs}}} S_{z y}
$$

where the contribution of $D_{z}$ was neglected. This is justified if $1 \ll \sigma_{0} / \epsilon_{\mathrm{GaAs}} \omega \ll 1 / \lambda_{D} k_{\mathrm{saw}}$, where $\lambda_{D}$ is the Debye length [19]. In our case $\sigma_{0} / \epsilon_{\mathrm{GaAs}} \omega \sim 10^{6}$ and the condition for disregarding $D_{z}$ is fulfilled. From Eqs. (1)(4) the current density due to the anomalous acoustoelectric effect in the center of the beam $z=L / 2$ calculates to

$$
j_{\mathrm{an}}(L / 2)=-\frac{\sigma_{0} e_{z 4}}{2 \epsilon_{\mathrm{GaAs}}}\left(\Pi_{z z z y} S_{z y}(L / 2)^{2}\right),
$$

where the mixed term $S_{z z}, S_{x y}$ in Eq. (1) equals 0, when integrated over one SAW period as the longitudinal and transversal SAW component are $\pi / 2$ phase shifted in time. The calculated current in dependence on $\phi$ is shown in the inset of Fig. 4(b). Equation (5) predicts a square dependence of the anomalous acoustoelectric current on the SAW amplitude. Using the measured input impedances of the two IDTs, it is possible to convert the rf power applied at the transducers into an estimate for the amplitude of the excited SAW [14]. Figure 4(b) shows the maximum of the anomalous acoustoelectric current component vs applied SAW amplitude. The curve follows the predicted behavior for not too small SAW amplitudes, which can be evaluated by fitting this section. The exponent we extract from this fit evaluates to $2.06 \pm 0.3$, which is in very good agreement with the expected value. From this fit we further estimate the size of $\Pi_{z z z y}$ to be on the order of $10^{5}$, which is 1 order of magnitude larger than observed for thin manganite films [19]. The minor deviations of the induced current for small SAW amplitudes suggests that the linear dependence of conductivity on strain might be an oversimplification. This can be due to different contributions like dislocations [14] or band deformation [22].

In summary, we have shown that shock waves can be generated in nanomechanical resonators. The resonators contain an electron gas, which we apply to probe the shock waves via the acoustoelectric effect.

We acknowledge support by the Deutsche Forschungsgemeinschaft (No. B1-487/3), an Air Force grant (No. AFOSR F49620-03-1-0420), and the National Science Foundation (No. NSF-NIRT 0708759).

*blick@engr.wisc.edu

[1] S. Zimmermann et al., Science 283, 1292 (1999).

[2] R.G. Knobel and A. N. Cleland, Nature (London) 424, 291 (2003).

[3] M. D. LaHaye et al., Science 304, 74 (2004).

[4] S. Savel'ev et al., Phys. Rev. B 75, 165417 (2007); S. Savel'ev et al., Phys. Rev. B 75, 165417 (2007); Fei Xue et al., Phys. Rev. B 76, 205302 (2007); Fei Xue et al., Phys. Rev. B 76, 064305 (2007).

[5] J.H. Davies, The Physics of Low-Dimensional Electron Systems: An Introduction (Cambridge University Press, Cambridge, England, 1998).

[6] R. H. Blick et al., Phys. Rev. B 62, 17103 (2000).

[7] E. M. Weig et al., Phys. Rev. Lett. 92, 046804 (2004).

[8] S. Debald, T. Brandes, and B. Kramer, Phys. Rev. B 66, 041301 (2002).

[9] M. Blencowe, Phys. Rep. 395, 159 (2004).

[10] M. D. Blumenthal et al., Nature Phys. 3, 343 (2007).

[11] A. Wixforth et al., Phys. Rev. B 40, 7874 (1989).

[12] F. W. Beil, A. Wixforth, and R.H. Blick, Proc. IEEE Sensors 1, 1285 (2002).

[13] F.W. Beil, R.H. Blick, and A. Wixforth, Physica (Amsterdam) 21E, 1106 (2004).

[14] F. W. Beil et al., Europhys. Lett. 76, 1207 (2006).

[15] F. W. Beil et al., Appl. Phys. Lett. 90, 043101 (2007).

[16] A. O. Govorov et al., Phys. Rev. B 62, 2659 (2000).

[17] J. M. Shilton et al., J. Phys. Condens. Matter 8, L531 (1996).

[18] V. I. Talyanskii et al., Phys. Rev. B 56, 15180 (1997).

[19] Y. Ilisavskii et al., Phys. Rev. Lett. 87, 146602 (2001).

[20] J. Kirschbaum et al., Appl. Phys. Lett. 81, 280 (2002).

[21] E. M. Höhberger et al., Appl. Phys. Lett. 82, 4160 (2003).

[22] C. Jagannath et al., Phys. Rev. B 34, 7027 (1986). 